East African Medical Journal Vol. 87 No. 6 June 2010

SUPPLEMENTAL OXYGEN FOR CAESAREAN SECTION UNDER SPINAL ANAESTHESIA

A.T. Adenekan, MBBS, DA, FWACS, Consultant Anaesthetist, A.F. Faponle, BSc., MBChB, FWACS, FMCA, Consultant Anaesthetist and E.A. Azebi, MBBS, DA, Department of Anaesthesia, Obafemi Awolowo University Teaching Hospital, PMB 5538, Ile-Ife, Nigeria

Request for reprints to: Dr. A. T. Adenekan, Department of Anaesthesia, Faculty of Clinical Sciences, College of Health Sciences, Obafemi Awolowo University, PMB 5538, 1le-Ife, Nigeria

\title{
SUPPLEMENTAL OXYGEN FOR CAESAREAN SECTION UNDER SPINAL ANAESTHESIA
}

\author{
A. T. ADENEKAN, A. F. FAPONLE and E. A. AZEBI
}

\begin{abstract}
Background: Routine administration of supplemental oxygen to parturients undergoing Caesarean section under spinal anaesthesia has been criticised in recent times. Objectives: To assess the need for routine supplementary oxygen in healthy women undergoing Caesarean section under spinal anaesthesia in resource challenged setting and establish the efficacy of administration of oxygen at $4 \mathrm{~L} / \mathrm{min}$.

Design: Simple randomized trial using sealed envelopes.

Setting: The Obafemi Awolowo University Teaching Hospital; a 580 bed hospital situated in Ile-Ife in South-western Nigeria.

Subjects: Seventy parturients with ASA physical status I or Il undergoing Caesarean section under spinal anaesthesia.

Main outcome measures: Outcome measures were arterial oxygen saturation $\left(\mathrm{SaO}_{2}\right)$ and Apgar scores at one and five minutes.

Results: The mean pre-induction arterial oxygen saturation in the two groups were similar. There was a statistically significant difference in the mean $\mathrm{SaO}_{2}$ at one minute between the two groups, with the control group being higher $(97.7 \% \pm 1.5 \%$ versus. $96.7 \% \pm 1.5 \%$; $\mathrm{p}$-value $=0.008$ ). The mean least $\mathrm{SaO}_{2}$ during surgery was also higher in the control group $(95.9 \% \pm 1.5 \%$ versus $94.9 \% \pm 2.0 \%$, $p$-value $=0.015)$. The Apgar score of the babies at one and five minutes for the study and control group were similar. Conclusion: Healthy parturients undergoing Caesarean section under spinal anaesthesia do well without supplemental oxygen; administration of supplemental oxygen from the common gas outlet of anaesthetic machine with the breathing circuit and standard anaesthetic facemask at $4 \mathrm{~L} / \mathrm{min}$ causes relative desaturation.
\end{abstract}

\section{INTRODUCTION}

Spinal anaesthesia has emerged as the preferred anaesthetic technique for Caesarean section despite its turbulent past $(1,2)$. Better maternal and foetal outcome has been firmly established with this technique compared to general anaesthesia $(2,3)$; and in resource challenged economies, cost reduction associated with its use provides extra relief. In many centres, supplemental oxygen is given routinely to parturients undergoing Caesarean section under spinal anaesthesia with the aim of increasing oxygen delivery to the foetus $(4,5)$. This practice has however come under criticism in recent times with reports showing that no significant improvement in foetal oxygenation occurs in mothers receiving $35-40 \%$ oxygen, and high fractional oxygen $(60 \%)$ could be potentially harmful to both mothers and foetus due to increase in free oxygen radical activity $(1,6)$.
Supplemental oxygen is often administered from the anaesthetic machine via the breathing circuit and a loosely fitting facemask. The efficacy of this method of oxygen delivery to parturients under spinal anaesthesia has not been previously studied in our centre. This study was performed to investigate the effects of supplemental oxygen administered via the anaesthetic machine and the breathing system on maternal arterial oxygen saturation, and Apgar scores of babies born to women having Caesarean section under spinal anaesthesia.

\section{MATERIALS AND METHODS}

Ethical approval was obtained from the Hospital Ethics and Research Committee, and written informed consent was obtained from the women recruited for the study. Sample size of seventy was arrived at using the equation forStudies Comparing Two Group 
Means (7) and a significance level of 0.05 , power of 0.9 and minimum expected differences between the two means of $2 \%$. Seventy women with ASA physical status I or II undergoing Caesarean section under spinal anaesthesia were included. Women requiring inhalational or intravenous general anaesthesia after subarachnoid block were excluded. Women with uncontrolled medical diseases and those for emergency Caesarean section on account of foetal distress were also excluded.

Spinal anaesthesia was given with the parturient either in sitting or lateral position. The intervertebral space (L4/5 or L3/4) was located and standard aseptic technique was observed. Spinal needle size $25 \mathrm{G}$ or $26 \mathrm{G}$ was inserted at the midline and correct placement was ascertained by free flow of cerebrospinal fluid after withdrawing the trocar. The desired volume of $0.5 \%$ heavy Bupivacaine Hydrochloride, 2.2 - $2.8 \mathrm{ml}$ was injected slowly. The women were returned to the supine position and made comfortable. A $15^{\circ}$ wedge was applied to the right buttock to prevent supine hypotension syndrome. The block height was ascertained satisfactory before surgery commenced. Monitoring of the vital signs continued with non-invasive blood pressure cycled at three minutes interval initially then at five minutes interval after the block was established. Verbal contact was maintained throughout the procedure. The women were randomly assigned to either the study group or control group using the envelope method with thirty-five women in each. The study group received supplemental oxygen at $4 \mathrm{~L} / \mathrm{min}$ from Datex Ohmeda ${ }^{\circledR}$ anaesthetic machine via the breathing circuit and a loosely fitting facemask after induction of spinal anaesthesia till the end of surgery and the control group breathed room air throughout the procedure.

Data collected include age, packed cell volume (PCV), indication for surgery, block height, complications, skin incision to delivery time, duration of surgery, and arterial oxygen saturation $\left(\mathrm{SaO}_{2}\right)$ pre-spinal and one minute after induction of spinal anaesthesia. The least $\mathrm{SaO}_{2}$ during surgery and the least during recovery period were also noted. The Apgar scores of the babies at one and five minutes after birth scored by the attending paediatrician were also collected.

Data were analysed with the Statistical Packages for the Social Sciences (SPSS) 16.0 for Windows Product. The results are presented as mean \pm standard deviation (SD) or percentages. Comparison was performed using the ANOVA procedure. A p-value $<0.05$ was considered significant.

\section{RESULTS}

Theindications for Caesarean section are summarised in Table 1. The characteristics of the two groups are shown in Table 2. The mean age, packed cell volume, block height, skin incision to delivery time, and duration of surgery of the two groups were similar. The mean pre-induction arterial oxygen saturation in the study and control groups were similar $(98.4 \% \pm$ $1.0 \%$ versus. $98.3 \% \pm 1.0 \% \mathrm{p}$-value $=0.524)$. However, there was a statistically significant difference in the mean arterial oxygen saturation at one minute between the two groups, with the control group (breathing room air) being higher $(97.7 \% \pm 1.5 \%$ versus. $96.7 \% \pm 1.8 \%$; p-value $=0.008)$. The mean least arterial oxygen saturation during surgery was also higher in the control group $(95.9 \% \pm 1.5 \%$ versus. $94.9 \% \pm 2.0 \%)$ and the difference was statistically significant $(p$-value $=0.015)$. The mean least arterial oxygen saturation during the recovery period for the study and the control group were $97.5 \% \pm 1.5 \%$ and $98.1 \% \pm 0.9 \%$ respectively, $p$-value $=0.046$. The least recorded $\mathrm{SaO}_{2}$ in the study and control groups were $91 \%$ and $92 \%$ respectively.

The Apgar score of the babies at one and five minutes for the study and control group were similar $(8.7 \pm 1.8$ versus. $8.3 \pm 1.8, \mathrm{p}$-value $=0.27 ; 9.8 \pm 0.9$ versus. $9.7 \pm 0.6$, $\mathrm{p}$-value $=0.75$ respectively). Two women in the study group had nausea and vomiting against none in the control group.

Table 1

Indication for Caesarean section

\begin{tabular}{lcc}
\hline Indication & Frequency & $(\%)$ \\
\hline Previous uterine scar & 23 & 32.9 \\
Failure to progress & 14 & 20.0 \\
Prolonged labour/ & & \\
Cephalopelvic disproportion & 9 & 12.9 \\
Abnormal/ unstable lie & 4 & 5.7 \\
Abnormal presentation & 3 & 4.3 \\
Foetal macrosomia & 3 & 4.3 \\
Multiple pregnancy & 3 & 4.3 \\
Obstructed labour & 3 & 4.3 \\
Post-datism & 2 & 2.9 \\
Others & 6 & 8.6 \\
\hline Total & 70 & 100 \\
\hline
\end{tabular}


Table 2

Characteristics of the two groups

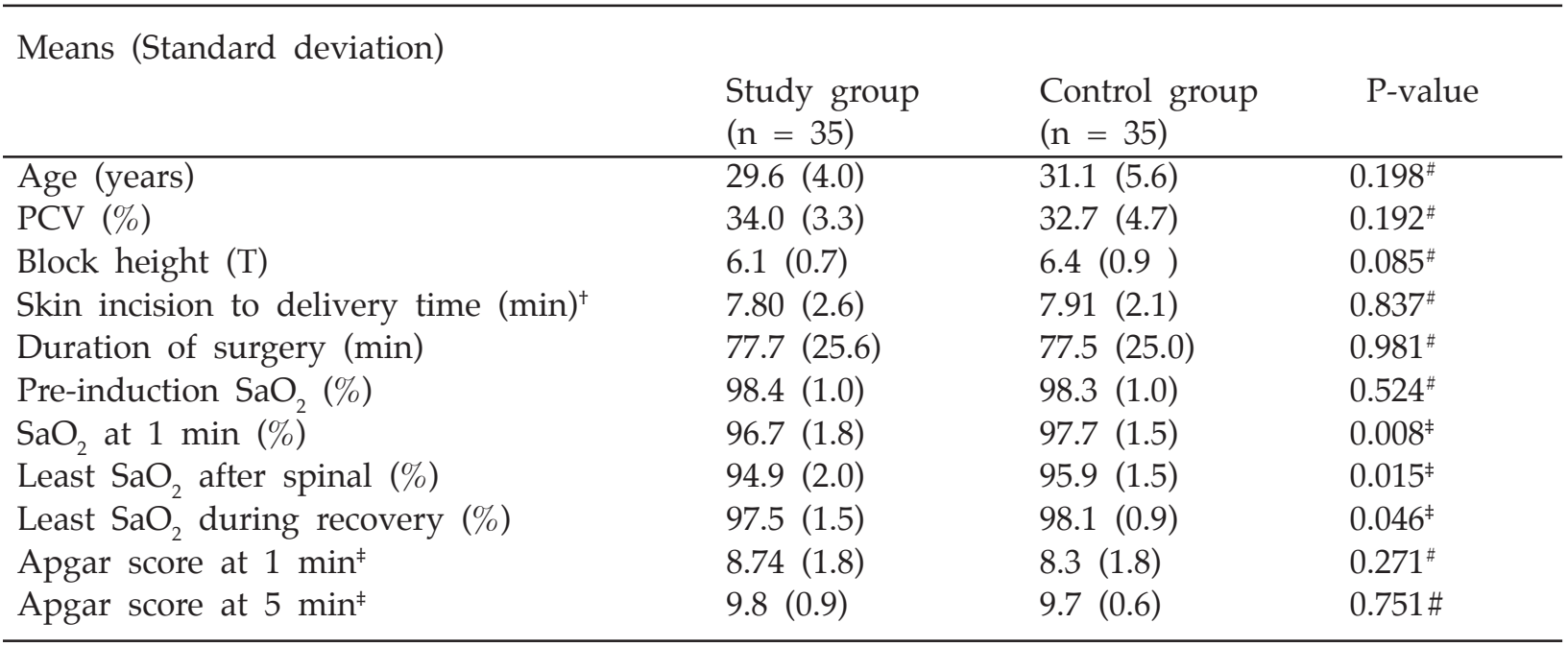

+ Apgar score/ Skin incision to delivery time of three second twin excluded from the analysis

₹ Statistically significant

\# Not significant

\section{DISCUSSION}

This study shows that supplemental oxygen administration from the anaesthetic machine via a closed circuit and facemask at $4 \mathrm{~L} / \mathrm{min}$ is associated with significant reduction in arterial oxygen saturation compared to a control group breathing room air. The difference which was first observed one minute post induction persisted till the immediate post operative period. However there was no difference in the neonatal outcome in both groups.

Routineadministration of supplemental oxygen to women undergoing Caesarean section under regional anaesthesia is practiced in many centres. This is aimed at improving foetal oxygenation and acid-base status, as well as increasing maternal oxygen reserve to enhance her ability to cope with stress of anaesthesia and surgery $(8,9)$. However, some publications $(1,6,10,11)$ have raised doubts about the benefit of this practise. Kelly et al (10) reported that parturients who received spinal anaesthesia had no maternal oxyhaemoglobin desaturation despite deterioration in respiratory mechanics. They observed that administration of $35 \%$ oxygen did not improve umbilical vein $\mathrm{PaO}_{2}$. Cogliano et al. (1) used $40 \%$ oxygen and made a similar observation in their report. Khaw et al. (6) in their study of effect of high $\mathrm{F}_{1} \mathrm{O}_{2}$ on maternal and foetal oxygenation and lipid peroxidation observed that administration of $60 \%$ $\mathrm{F}_{1} \mathrm{O}_{2}$ to parturients undergoing spinal anaesthesia resulted in a modest increase in umbilical vein $\mathrm{PaO}_{2}$ with no difference in neonatal outcome. Moreover, administration of a high $\mathrm{F}_{1} \mathrm{O}_{2}$ was associated with increased free radical activity in both the mother and foetus which may be potentially harmful. Similar findings were reported by Singh et al (11).
While the controversies continue, safety and wellbeing of mother and child must remain the primary consideration of anaesthetists and obstetricians.

This study differs from previous reports which were done in well resourced settings with standardised oxygen delivery devices used for the studies. Cogliano et al (1) and Crosby and Halpem (9) used variable performance masks (medium concentration oxygen devices) whereas Khaw et al (6) and Singh et al (11) used venti mask. However, constrains with regular supply of disposables in developing nations, has forced many clinicians to continue to use reusable equipment and accessories. Reusable breathing circuits and facemasks were used to administer supplemental oxygen in this study.

Using standard medium concentration oxygen mask with a flow rate of $4 \mathrm{~L} / \mathrm{min}\left(\mathrm{F}_{1} \mathrm{O}_{2}\right.$ of 0.4$)$, Cogliano et al (1) observed that some women complained about the smell of the facemask and gas, felt claustrophobic, had difficulty in getting enough air, and were unable to communicate. Although, comfort, communication and smell of the gas delivery system were not assessed in our study, these effects may explain the relative desaturationobserved in thestudy groupinthisstudy. Two parturients who had nausea and vomiting observed in this study were in the study group. Although, this does not amount to statistical significance, the possible role of the smell of the gas and mask cannot be excluded.

In high risk parturients who undoubtedly will benefit from supplemental oxygen under regional anaesthesia the use of nasal prongs for delivery of supplemental oxygen at $2-4 \mathrm{~L} / \mathrm{min}$ has been found to be better tolerated and advocated (1). In settings where these disposable devises are not available, improvised nasal prong using either a naso-gastric tube or an infusion giving set may be an alternative. 
A case has been made for the administration of supplemental oxygen from a separate and dedicated source on safety grounds to obviate critical incidents and adverse outcomes that have been associated with the use common gas outlet of the anaesthetic machine $(5,12)$. However, cost implication may be a major setback to theimplementation of this in resource challenged economies. An additional safety practice to ensure the fresh gas flow is reconnected before a general anaesthetic can be given suggested by Edsell and Erasmus (12) appears to be a more practical option. The proximal end of the breathing system (the fresh gas flow connection) is attached to the distal end of the circuit whenever supplementary oxygen is being given to the patient from the common gas outlet. In order for the distal end to be connected to a facemask (tostart pre-oxygenation), the proximal end must first be disconnected. This acts as a reminder to the anaesthetist to reconnect the breathing circuit to the common gas outlet. None of the women in this study developed maternal hypoxaemia defined as $\mathrm{SaO}_{2}$ less than $90 \%$ corresponding to an arterial oxygen tension less than $7.9 \mathrm{kPa}(11)$. The recorded mean least $\mathrm{SaO}_{2}$ during surgery in the control group was $95.9 \% \pm 1.5 \%$ with a range of $92-99 \%$.

This is lower than the least recorded $\mathrm{SaO}_{2}(94 \%)$ by Cogliano et al (1) in their study. All the babies in this study were well enough to leave the recovery room with their mothers and none required admission to the Special Care Baby Unit.

One major limitation of our study is that maternal arterial and foetal umbilical arterial and venous blood gas analysis were not performed. These are not routinely available in low resource centres. At clinically acceptable levels of arterial oxygenation, the $\mathrm{O}_{2}$ saturation recorded by pulse oximeters differs by $<3 \%$ from the actual $\mathrm{SaO}_{2}(13,14)$. However, satisfactory maternal $\mathrm{SaO}_{2}$ may not translate to normal foetal umbilical blood gases as there may be problem with the maternal-foetal gas exchange. Also, uterine incision-delivery interval is not routinely recorded in our centre and was not included in this study. However, skin incision and uterine incision to delivery intervals have been found not to significantly contribute to neonatal outcome when corrected for labour complications, infant weight, type of Caesarean delivery, and type of anaesthesia (15). Skin incision delivery intervals and Apgar scores were comparable in both groups in this study.

In conclusion, this study shows that healthy parturients undergoing Caesarean section underspinal anaesthesia and their babies do well without supplemental oxygen. Administration of supplemental oxygen to parturients from the common gas outlet of anaesthetic machine with the breathing circuit and standard anaesthetic facemask at $4 \mathrm{~L} / \mathrm{min}$ causes relative desaturation and should be avoided. In centres where continuous pulse oximetry can be done, it may be logical to administer supplemental oxygen to parturients only when necessary. In maternal or foetal comprise, supplemental oxygen is beneficial and should not be spared. However, the source and delivery systems should be carefully selected to avoid potential disasters that can complicate this practice.

\section{REFERENCES}

1. Cogliano, M.S., Graham, A.C. and Clark, V.A. Supplementary oxygen administration for elective Caesarean section under spinal anaesthesia. Anaesthesia. 2002; 57: 66-69.

2. Morgan, P. Spinal anaesthesia in obstetrics - review article. Can. J. Anaesth. 1995; 42:1145-1163.

3. Ilori, I.U., Akpan, S.G. and Eshiet, A.I. The effect of anaesthetic technique on the apgar score of neonates delivered by emergency Caesarean section. Afr. J. Anaesth. Inten. Care. 2005; 6: 5-7.

4. Ogunbiyi, O.A., Mato, C.N. and Isong, E.C. Oxygen saturation after spinal anaesthesia. Any need for supplemental oxygen? Afr. J. Anaesth. Inten. Care. 2003; 4: 5-6.

5. Stone, A.G.H. and Howell, P.R. Use of the common gas outlet for the administration of supplemental oxygen during Caesarean section under regional anaesthesia. Anaesthesia. 2002; 57: 690-709.

6. Khaw, K.S., Wang, C.C., Kee, W.D.N., et al. Effects of high inspired oxygen fraction during elective Caesarean section under spinal anaesthesia on maternal and fetaloxygenation and lipid peroxidation. Br. J. Anaesth. 2002; 88: 18-23.

7. Eng, J.Samplesize estimation: How many individuals should be studied? Radiology. 2003; 227: 309 - 313.

8. Jordan, M.J. Women undergoing Caesarean section under regional anaesthesia should routinely receive supplemental surgery. Int. J. Obstet. Anesth. 2002; 11: 282-285.

9. Crosby, E.T. and Halpem, S.H.Supplemental maternal oxygen therapy during Caesarean section under epidural anaesthesia: a comparison of nasal prongs and facemask. Can. J. Anaesth. 1992; 39: 313-316.

10. Kelly, M.C., Fitzpatrick, K.T. and Hill, D.A. Respiratory effects of spinal anaesthesia for Caesarean section. Anaesthesia. 1996; 51: 1120-1122.

11. Singh, V., Hooda, S., Dahiya, K. and Sharma, R. Effect of different inspired oxygen concentrations during caesarean section under spinal anaesthesia on maternal and foetal oxygenation and lipid peroxidation. Bombay Hosp. J. 2006; 48: 561-566.

12. Edsell, M.E.G. and Erasmus, P.D. Use of the common gas outlet for supplementary oxygen during Caesarean section (Correspondence). Anaesthesia. 2005; 60: 1152.

13. Wahr, J.A. and Tremper, K.K. Noninvasive oxygen monitoring techniques. Crit. Care Clin. 1995; 11: 199217.

14. Severinghaus, J.W. and Kelleher, J.F. Recent developments in pulse oximetry. Anesthesiology. 1992; 76: 1018-1038.

15. Andersen, H.F., Auster, G.H., Marx, G.F. and Merkatz, I.R. Neonatal status in relation to incision intervals, obstetric factors, and anaesthesia at caesarean delivery. Am. J. PerinatoI. 1987; 4: 279-283. 